TRANSACTIONS OF THE

AMERICAN MATHEMATICAL SOCIETY

Volume 353, Number 3, Pages 893-905

S 0002-9947(00)02609-X

Article electronically published on November 8, 2000

\title{
BICANONICAL PENCIL OF A DETERMINANTAL BARLOW SURFACE
}

\author{
YONGNAM LEE
}

\begin{abstract}
In this paper, we study the bicanonical pencil of a Godeaux surface and of a determinantal Barlow surface. This study gives a simple proof for the unobstructedness of deformations of a determinantal Barlow surface. Then we compute the number of hyperelliptic curves in the bicanonical pencil of a determinantal Barlow surface via classical Prym theory.
\end{abstract}

\section{InTRODUCTION}

The first example of a surface of general type with $p_{g}=q=0, K^{2}=1$ was constructed by Godeaux as a quotient of a quintic in $\mathbb{P}^{3}$ under a free $\mathbb{Z}_{5}$-action. For the generic such Godeaux surface $X$, it is easily computed that $h^{1}\left(T_{X}\right)=8$ and $h^{2}\left(T_{X}\right)=0$. In the eight-dimensional family of Godeaux surfaces, there is a fourdimensional subfamily for which the quintic is symmetric determinantal. This family was studied by Catanese in [C1]. We call these surfaces determinantal Godeaux surfaces. Again, inside this four-dimensional family, there is a two-dimensional subfamily for which the $\mathbb{Z}_{5}$-action on the (symmetric determinantal) quintic extends to an action of the dihedral group $D_{5}$. By twisting this action, Barlow constructed the first example of a simply connected surface of general type with $p_{g}=0$ in $[\mathrm{Ba}$. In fact, as Barlow remarks, her twisting works for the entire two-dimensional family mentioned above, giving a two-dimensional family of simply connected surfaces which we call determinantal Barlow surfaces.

Recently, in CL, Catanese and Le Brun proved $h^{2}\left(T_{B}\right)=0$ for a general determinantal Barlow surface in view of applications to a differential geometry. The purpose of this paper is to present a geometric proof. The outline of the proof is as follows.

A numerical Godeaux surface $X$ (surface of general type with $p_{g}=0, K^{2}=$ 1) has a bicanonical pencil. We consider the case in which all members of this pencil are semistable curves of genus four. This pencil then gives a rational curve $\mathbb{P}_{X}^{1}$ in $\overline{\mathcal{M}_{4}}$, the Deligne-Mumford compactification of the moduli space of curves of genus four. In the case in which $X$ is a $D_{5}$-invariant determinantal Godeaux

Received by the editors October 15, 1997 and, in revised form, June 3, 1999.

2000 Mathematics Subject Classification. Primary 14J10, 14J29.

The author would like to express his appreciation to professor Herb Clemens for valuable suggestions that made this work possible. Also the author would like to thank Professor Fabrizio Catanese for the use of his approach and the results in his recent preprint (written with Pignatelli) to solve the problem of hyperelliptic curves. Finally, the author would like to thank the referee for many interesting suggestions and corrections. Most of this work is the part of a Ph.D. thesis submitted to the University of Utah in 1997, and is partially supported by the Korea Institute for Advanced Study. 
surface, Barlow's construction distinguishes, for each $\{C\} \in \mathbb{P}_{X}^{1}$, a cardinality-four group of unbranched double covers of $C$. Letting $\mathcal{U}_{X}$ denote a sufficiently small analytic neighborhood of $\mathbb{P}_{X}^{1} \subseteq \overline{\mathcal{M}_{4}}$, we lift $\mathbb{P}_{X}^{1} \subseteq \mathcal{U}_{X}$ to $\mathbb{P}_{Z}^{1} \subseteq \mathcal{U}_{Z}$, a small analytic neighborhood in $\overline{\mathcal{M}_{7, i}}=\overline{\mathcal{R}_{7}}$, the (suitably compactified) space of pairs $(\tilde{C}, i)$, where $\tilde{C}$ has genus seven and $i$ is a fixpoint-free involution. Then Barlow's twisting action $a$ takes $\mathcal{U}_{Z}$ isomorphically to $\mathcal{U}_{Z}^{\prime}$ in $\overline{\mathcal{M}_{7, i}}$, and gives a commutative diagram:

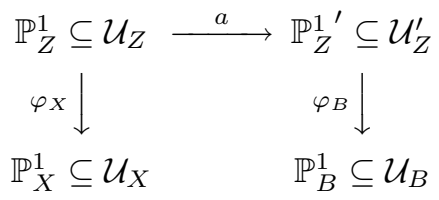

We reduce the problem of computing $H^{2}\left(T_{S}\right)$ for a numerical Godeaux surface $S$ to the problem of computing $H^{1}\left(N_{\mathbb{P}_{S}^{1}} \mid \overline{\mathcal{M}_{4}}\right)$. Then we compute $N_{\mathbb{P}_{X}^{1}} \mid \overline{\mathcal{M}_{4}}=\mathcal{O}(1)^{4}+$ $\mathcal{O}(-1)^{4}$ for a general $D_{5}$-invariant determinantal Godeaux surface $X$. By a careful study of the degeneracy locus of $\varphi_{X}$, we prove $N_{\mathbb{P}_{Z}^{1}} \mid \overline{\mathcal{M}_{7, i}}=\mathcal{O}^{4}+\mathcal{O}(-1)^{4}$ or $\mathcal{O}(1)^{2}+$ $\mathcal{O}(-1)^{2}+\mathcal{O}(-1)^{4}$. Since $a$ preserves the normal bundle and the degrees do not decrease under the finite morphism $\varphi_{B}$, we obtain that $H^{2}\left(T_{B}\right)=0$.

Finally, the computation of the numerical invariants of $\mathbb{P}_{B}^{1}$ employed in the above argument make it possible to compute the number of hyperelliptic curves in $\mathbb{P}_{B}^{1}$ following a line of argument introduced by Catanese. The new ingredient introduced here is to use a Prym construction to reduce this computation to computing the numerical invariants of certain rational curves in $\overline{\mathcal{M}_{3}}$.

\section{Bicanonical pencil of a Godeaux surface}

Let $(x, y, z, w)$ be the coordinates of $\mathbb{P}^{3}$, and consider a natural $\mathbb{Z}_{5}$-action on $\mathbb{P}^{3}$ via

$$
(x, y, z, w) \rightarrow\left(\epsilon x, \epsilon^{2} y, \epsilon^{3} z, \epsilon^{4} w\right)
$$

where $\epsilon$ is a primitive 5 -th root of unity. The $\mathbb{Z}_{5}$-invariant quintics $\Sigma$ without fixed points form an irreducible eight-dimensional family $\mathcal{M}$ [Mi] :

$$
\begin{aligned}
x^{5}+y^{5}+z^{5}+w^{5} & +t_{1} x^{3} z w+t_{2} x y w^{3}+t_{3} x y^{3} z+t_{4} y z^{3} w \\
& +t_{5} x^{2} y z^{2}+t_{6} y^{2} z w^{2}+t_{7} x^{2} y^{2} w+t_{8} x z^{2} w^{2} .
\end{aligned}
$$

Since the four fixed points of the $\mathbb{Z}_{5}$-action are not in $\Sigma$, the quotient $X=\Sigma / \mathbb{Z}_{5}$ is a smooth minimal projective surface of general type, with $p_{g}=q=0, K^{2}=1$, and $\pi_{1}(X)=\mathbb{Z}_{5}$. We call these surfaces Godeaux surfaces. For a Godeaux surface, the bicanonical pencil (resp. the tricanonical linear system) is given by $H^{0}\left(2 K_{\Sigma}\right)^{\mathbb{Z}_{5}}$ (resp. $\left.H^{0}\left(3 K_{\Sigma}\right)^{\mathbb{Z}_{5}}\right)$. Then $H^{0}\left(2 K_{\Sigma}\right)^{\mathbb{Z}_{5}}$ is generated by $x w, y z$, and $H^{0}\left(3 K_{\Sigma}\right)^{\mathbb{Z}_{5}}$ is generated by $x y^{2}, x^{2} z, y w^{2}, z^{2} w \cdot\left|2 K_{\Sigma}\right|^{\mathbb{Z}_{5}}$ has 20 base points on $\Sigma$ :

$$
\left(1,0,-\epsilon^{i}, 0\right),\left(1,-\epsilon^{i}, 0,0\right),\left(0,-\epsilon^{i}, 0,1\right),\left(0,0,-\epsilon^{i}, 1\right)
$$

Also $\left|3 K_{\Sigma}\right|^{\mathbb{Z}_{5}}$ has 10 base points on $\Sigma$ : $\left(1,0,0,-\epsilon^{i}\right),\left(0,1,-\epsilon^{i}, 0\right)$.

Example 1. Consider a smooth quintic $\Sigma: x^{5}+y^{5}+z^{5}+w^{5}=0$ and a pencil $D: \lambda x w-\mu y z$ for $(\lambda, \mu) \in \mathbb{P}^{1}$. If $\lambda=0$ or $\mu=0$, then $D=D_{1}+D_{2}$ and $D_{1} \cdot D_{2}$ are five base points of $\left|3 K_{\Sigma}\right|^{\mathbb{Z}_{5}}$. By an easy computation, other singular members of $D \cap \Sigma$ are provided by $\mu^{5}+1=0$. 
Consider $X=\Sigma / \mathbb{Z}_{5}$ and the bicanonical pencil of $X$. After blowing up at the four base points of $\left|2 K_{X}\right|$, there is a fibration $f: S \rightarrow \mathbb{P}^{1}$ and each fiber of $f$ is a stable curve of genus four. There are two types of singular fibers. Two singular fibers $(\lambda=0$ or $\mu=0)$ are $C_{1}+C_{2}$ where $C_{1}, C_{2}$ are smooth curves of genus two. The intersection point between $C_{1}$ and $C_{2}$ is exactly one of the base points of $\left|3 K_{X}\right|$ [C2, $\mathrm{Mi}$ ] or [Re. Five other singular fibers are $C_{1}+C_{2}$, where each $C_{i}$ is $\mathbb{P}^{1}$ with $C_{1} . C_{2}=5$ and each intersection is simple.

By a tedious computation, if we add $t x^{3} z w$ to the equation of $\Sigma$ for general $t$, then these 5 singular fibers deform to 25 singular fibers with an ordinary quadratic singularity.

According to the irreducibility of the moduli space of Godeaux surfaces, and by Example 1 we can conclude the following:

Lemma 2. Choose a generic element $X$ in the moduli space of Godeaux surfaces. Then $\left|2 K_{X}\right|$ has no base components, and it has four simple base points. Let $p$ : $S \rightarrow X$ be the blow up of the 4 base points of $\left|2 K_{X}\right|$. Then each fiber of $f: S \rightarrow \mathbb{P}^{1}$ is a stable curve of genus four, and it has at most one node.

Let $C$ be a general element of the bicanonical pencil of a Godeaux surface. Consider the following commutative diagram:

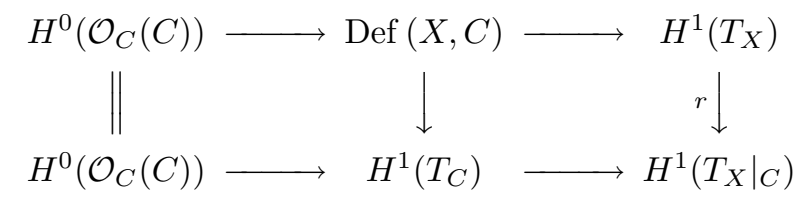

$0 \rightarrow H^{1}\left(T_{X} \otimes I_{C}\right) \rightarrow H^{1}\left(T_{X}\right) \stackrel{r}{\longrightarrow} H^{1}\left(\left.T_{X}\right|_{C}\right) \rightarrow H^{2}\left(T_{X} \otimes I_{C}\right) \rightarrow H^{2}\left(T_{X}\right) \rightarrow 0$.

In the above diagram, $\operatorname{Def}(X, C)$ means the first order infinitesimal deformation of the pair $(X, C)$, that is the first hypercohomology group of the map

$$
T_{X} \rightarrow N_{C \mid X}
$$

$\operatorname{Def}(X, C)=H^{1}\left(T_{X, C}\right)$ where $T_{X, C}$ is the sheaf of vector fields (also it is called $T_{X}(-\log C)$ by other authors) whose restriction to $C$ lies in $T_{C}$. And the map $H^{1}\left(T_{X, C}\right) \rightarrow H^{1}\left(T_{C}\right)$ is induced by the restriction. Since we have $H^{1}\left(\mathcal{O}_{C}(C)\right)=$ $H^{0}\left(\mathcal{O}_{C}\left(K_{X}\right)\right)^{*}=0\left(p_{g}(X)=q(X)=0\right)$, surjection of the map $\operatorname{Def}(X, C) \rightarrow$ $H^{1}\left(T_{C}\right)$ is the same as the surjection of $H^{1}\left(T_{X}\right) \rightarrow H^{1}\left(\left.T_{X}\right|_{C}\right)$. According to the Hirzebruch-Riemann-Roch theorem

$$
\chi\left(T_{X} \otimes I_{C}\right)=\chi\left(T_{X}\left(-2 K_{X}\right)\right)=-10 \chi\left(\mathcal{O}_{X}\right)+10 K_{X}^{2}=0 .
$$

So we have $h^{1}\left(T_{X}\left(-2 K_{X}\right)\right)=h^{2}\left(T_{X}\left(-2 K_{X}\right)\right)$. In particular, for a Godeaux surface $H^{2}\left(T_{X}\right)=H^{2}\left(T_{\Sigma}\right)^{\mathbb{Z}_{5}}=0$ where $\Sigma$ is a $\mathbb{Z}_{5}$-invariant smooth quintic in $\mathbb{P}^{3}$. And we have $H^{2}\left(T_{X}\left(-2 K_{X}\right)\right)=H^{0}\left(\Omega_{X}\left(3 K_{X}\right)\right)=H^{0}\left(\Omega_{\Sigma}(3)\right)^{\mathbb{Z}_{5}} \cdot H^{0}\left(\Omega_{\Sigma}(3)\right)^{\mathbb{Z}_{5}}$ can be computed in the following way:

Consider two exact sequences

$$
\begin{aligned}
0 & \left.\rightarrow \mathcal{O}_{\Sigma}(-2) \rightarrow \Omega_{\mathbb{P}^{3}}(3)\right|_{\Sigma} \rightarrow \Omega_{\Sigma}(3) \rightarrow 0 \\
0 & \left.\rightarrow \Omega_{\mathbb{P}^{3}}(3)\right|_{\Sigma} \rightarrow \mathcal{O}_{\Sigma}(2)^{4} \rightarrow \mathcal{O}_{\Sigma}(3) \rightarrow 0 .
\end{aligned}
$$


From the first sequence, $H^{0}\left(\Omega_{\Sigma}(3)\right) \cong H^{0}\left(\left.\Omega_{\mathbb{P}^{3}}(3)\right|_{\Sigma}\right)$. According to the second sequence,

$$
H^{0}\left(\Omega_{\Sigma}(3)\right)=k e r:\left(H^{0}\left(\mathcal{O}_{\mathbb{P}^{3}}(2)\right)\right)^{4} \rightarrow H^{0}\left(\mathcal{O}_{\mathbb{P}^{3}}(3)\right) .
$$

The map $\left.H^{0}\left(\mathcal{O}_{\mathbb{P}^{3}}(2)\right)\right)^{4} \rightarrow H^{0}\left(\mathcal{O}_{\mathbb{P}^{3}}(3)\right)$ is given by the matrix $(x, y, z, w)$. By an easy computation, $h^{0}\left(\Omega_{\Sigma}(3)\right)=20$. After considering the $\mathbb{Z}_{5}$-action on each term we obtain $h^{0}\left(\Omega_{\Sigma}(3)\right)^{\mathbb{Z}_{5}}=4$. It is generated by $\left(y^{2},-x y, 0,0\right),\left(x z, 0,-x^{2}, 0\right)$, $\left(0, w^{2}, 0,-y w\right)$ and $\left(0,0, z w,-z^{2}\right)$ in $H^{0}\left(\mathcal{O}_{\mathbb{P}^{3}}(2)\right)^{4}$. Therefore we obtain the following lemma.

Lemma 3. Let us call a stable curve $C$ of genus four a "Godeaux curve" if it occurs as a fiber of the bicanonical pencil for a smooth Godeaux surface. Then the family of Godeaux curves has dimension five.

Lemma 3 can also be easily obtained by the following argument: Let $h=0$ be a $\mathbb{Z}_{5}$-invariant quintic equation. Then consider the 2-parameter family

$$
g_{(\lambda, \mu)}=h+(\lambda x w-\mu y z)\left(\mathbb{Z}_{5} \text {-invariant cubic }\right)=0 .
$$

Since the bicanonical pencil of Godeaux surface comes from the pencil $\lambda x w-\mu y z$, $h=0$ and $g_{(\lambda, \mu)}=0$, both contain the same curve cutting by $\lambda x w-\mu y z=0$. The dimension of the family of $\mathbb{Z}_{5}$-invariant cubics is four, so each curve of the bicanonical pencil belongs to a four-dimensional family of Godeaux surfaces.

Consider the Deligne-Mumford compactification of the moduli space of curves of genus four, $\overline{\mathcal{M}_{4}}$. In this paper, $\overline{\mathcal{M}_{4}}$ is mostly a moduli functor instead of a moduli space, because we are usually interested in numerical data of the pull back to the pencil. The Hodge class $\lambda$ and the boundary classes $\delta_{0}, \delta_{1}, \delta_{2}$ are the standard basis of Pic $\overline{\mathcal{M}_{4}} \otimes \mathbb{Q}$; see $[\mathrm{AC}] . f: S \rightarrow \mathbb{P}^{1}$ induces a morphism $\tilde{q}: \mathbb{P}^{1} \rightarrow \overline{\mathcal{M}}_{4} . f_{*} \omega_{S / \mathbb{P}^{1}}$ is locally free of rank four and of degree,

$$
\chi\left(\mathcal{O}_{S}\right)-\left(g\left(\mathbb{P}^{1}\right)-1\right)(g(C)-1)=4
$$

by the Leray spectral sequence and the Riemann-Roch theorem. Consider this rank four vector bundle $f_{*} \omega_{S / \mathbb{P}^{1}}$. By a classical lemma of Grothendieck $f_{*} \omega_{S / \mathbb{P}^{1}}=$ $\sum_{i=1}^{4} \mathcal{O}_{\mathbb{P}^{1}}\left(a_{i}\right)$. According to the semi-positiveness theorem of curves (Theorem of Fujita in $[\mathrm{Fu}]$ ), every locally free quotient of $f_{*} \omega_{S / \mathbb{P}^{1}}$ is of nonnegative degree. Furthermore, the number of summands of $f_{*} \omega_{S / \mathbb{P}^{1}}$ isomorphic to $\mathcal{O}_{\mathbb{P}^{1}}$ is $q(S)-$ $g\left(\mathbb{P}^{1}\right)=0$; see [B3]. So we have

$$
f_{*} \omega_{S / \mathbb{P}^{1}}=\mathcal{O}_{\mathbb{P}^{1}}(1)^{4} .
$$

Then, by an easy computation (consider Example 1, our computation is on the moduli functor; see [AC]), we obtain the following intersection data of the pull back to the pencil:

Lemma 4. Let $f: S \rightarrow \mathbb{P}^{1}$ be the bicanonical pencil of a general Godeaux surface. Then $\lambda \cdot \mathbb{P}^{1}=4, \delta_{0} \cdot \mathbb{P}^{1}=25, \delta_{1} \cdot \mathbb{P}^{1}=0$ and $\delta_{2} \cdot \mathbb{P}^{1}=2$.

According to the deformations of morphisms of curves [ $\mathrm{KO}, \mathrm{II} .1]$,

$$
\operatorname{dim} \operatorname{Hom}_{[\tilde{q}]}\left(\mathbb{P}^{1}, \overline{\mathcal{M}}_{4}\right) \geq-K_{\overline{\mathcal{M}}_{4}} \cdot \mathbb{P}^{1}+9 \chi\left(\mathcal{O}_{\mathbb{P}^{1}}\right) .
$$

Since $\delta_{1} \cdot \mathbb{P}^{1}=0$, we have the following intersection number with $K_{\overline{\mathcal{M}}_{4}}$ :

$$
K_{\overline{\mathcal{M}}_{4}} \cdot \mathbb{P}^{1}=\left(13 \lambda-2 \delta_{0}-2 \delta_{2}\right) \cdot \mathbb{P}^{1}=-2[\mathrm{HM}] .
$$

The above computation gives $\operatorname{dim} \operatorname{Hom}_{[\tilde{q}]}\left(\mathbb{P}^{1}, \overline{\mathcal{M}}_{4}\right)$ at least 11 . 
Lemma 5. Consider the deformation space of $S$ with the bicanonical pencil. Then $\operatorname{dim} \operatorname{Hom}_{[\tilde{q}]}\left(\mathbb{P}^{1}, \overline{\mathcal{M}}_{4}\right)-3 \leq$ dimension of the deformation space of $S$ with the bicanonical pencil.

Proof. Regard $\overline{\mathcal{M}_{4}}$ as a moduli functor, then there is a universal space over $\overline{\mathcal{M}_{4}}$. According to a commutative diagram:

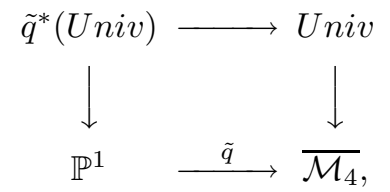

deformation of $\tilde{q}$ induces a deformation of $S$ with the bicanonical pencil $D$ (since the surface $S$ has $q=0, D$ remains $2 K$ ). Choose two different $\mathbb{P}^{1}$ in $\overline{\mathcal{M}_{4}}$. Because $\left|2 K_{S}\right|$ is a pencil, the pull back of universal spaces is not isomorphic. So $\operatorname{dim} \operatorname{Hom}_{[\tilde{q}]}\left(\mathbb{P}^{1}, \overline{\mathcal{M}}_{4}\right)-3 \leq$ dimension of the deformation space of $(S, D)$.

Since the dimension of the deformation space of the pair $(S, D)$ is eight, we have

$$
\operatorname{dim} \operatorname{Hom}_{[\tilde{q}]}\left(\mathbb{P}^{1}, \overline{\mathcal{M}}_{4}\right)=11 .
$$

Also it implies $H^{1}\left(\mathbb{P}^{1}, \tilde{q}^{*} T_{\overline{\mathcal{M}_{4}}}\right)=0[\mathrm{Ko}$, II.1].

Theorem 6. Let $\tilde{q}: \mathbb{P}^{1} \rightarrow \overline{\mathcal{M}_{4}}$ be the bicanonical pencil of a Godeaux surface. Then

$$
\tilde{q}^{*} T_{\overline{\mathcal{M}_{4}}}=\mathcal{O}(-1)^{4}+\mathcal{O}(1)^{4}+\mathcal{O}(2) .
$$

Proof. Since a five-dimensional subfamily of curves in $\overline{\mathcal{M}_{4}}$ comes from Godeaux surfaces by Lemma 3,

$$
\tilde{q}^{*} T_{\overline{\mathcal{M}_{4}}}=\sum_{i=0}^{4} \mathcal{O}\left(a_{i}\right)+\sum_{j=1}^{4} \mathcal{O}\left(b_{j}\right)
$$

where $a_{i} \geq 0$ and $b_{j}<0$. Since $h^{1}\left(\mathbb{P}^{1}, \tilde{q}^{*} T_{\overline{\mathcal{M}_{4}}}\right)=0$, so all $b_{j}=-1 .-K_{\overline{\mathcal{M}_{4}}} \cdot \mathbb{P}^{1}=2$ implies that $a_{0}+\cdots+a_{4}=6$.

Let $\overline{\mathcal{D}_{4}}$ be the closure of this five-dimensional subfamily of curves in $\overline{\mathcal{M}_{4}}$. Then $\tilde{q}^{*} T_{\overline{\mathcal{D}_{4}}}$ should be ample, because if we fix one element $[C] \in \overline{\mathcal{D}_{4}}$ there is a four dimensional family $\mathcal{V}$ of Godeaux surfaces that contain $[C]$, and $\mathbb{P}^{1} \times \mathcal{V} \rightarrow \overline{\mathcal{D}_{4}}$ is dominant [Ko, II.3]. This argument shows that all $a_{i}$ are at least 1 . So we obtain the conclusion that one of them equals 2 , while others equal 1 .

Corollary 7. Let $\tilde{q}: \mathbb{P}^{1} \rightarrow \overline{\mathcal{M}_{4}}$ be the pencil of a Godeaux surface. Then for every $x_{1}, x_{2} \in \tilde{q}\left(\mathbb{P}^{1}\right)$, there is a 1-cycle $\sum e_{i} E_{i}$ whose irreducible components are rational curves such that

$$
\tilde{q}_{*}\left(\mathbb{P}^{1}\right) \approx_{e} \sum e_{i} E_{i}
$$

where $\sum e_{i} \geq 2$ and $x_{1}, x_{2} \in S u p p \sum e_{i} E_{i}\left(\approx_{e}\right.$ means effective algebraic equivalence [Ko, II.4]).

Proof. $\operatorname{dim} \operatorname{Hom}_{[\tilde{q}]}\left(\mathbb{P}^{1}, \overline{\mathcal{D}_{4}}\right)=\operatorname{dim} \operatorname{Hom}_{[\tilde{q}]}\left(\mathbb{P}^{1}, \overline{\mathcal{M}_{4}}\right)=11=2 \operatorname{dim} \overline{\mathcal{D}_{4}}+1$. According to the theorem of Bend and Break, we obtain this corollary [Ko, II.5]. 


\section{BiCANONICAL PENCIL OF A DETERMinANTAL BARLOW SURFACE}

There is a four-dimensional subfamily of the moduli space of Godeaux surfaces that comes from $\mathbb{Z}_{5}$-invariant symmetric determinantal quintics. We will call these surfaces determinantal Godeaux surfaces. These surfaces were studied by Catanese in [C1. If $\Sigma$ is a symmetric determinantal quintic in $\mathbb{P}^{3}$, then $\Sigma$ has an even set of 20 nodes. An even set of nodes means that there is a double cover $\sigma: Y \rightarrow \Sigma$ branched over nodes. Let $\tilde{\Sigma}$ be the minimal resolution of $\Sigma$, and $\Delta$ be the sum of the twenty $(-2)$-curves. The author proves $H^{0}\left(\tilde{\Sigma}, 3 K_{\tilde{\Sigma}}-\Delta\right)=0, H^{2}\left(T_{\tilde{\Sigma}}\right)=0$ in L1 $\S 3.2]$, by using the arguments of Beauville in [B1] and [B2].

Let $\left(y_{1}, y_{2}, y_{3}, y_{4}\right)$ be the coordinates of $\mathbb{P}^{3}$, and consider a $D_{5}=\langle b, a\rangle$ action on $\mathbb{P}^{3}$ via

$$
\begin{aligned}
& b:\left(y_{1}, y_{2}, y_{3}, y_{4}\right) \rightarrow\left(\epsilon y_{1}, \epsilon^{2} y_{2}, \epsilon^{3} y_{3}, \epsilon^{4} y_{4}\right) \\
& a:\left(y_{1}, y_{2}, y_{3}, y_{4}\right) \rightarrow\left(y_{4}, y_{3}, y_{2}, y_{1}\right)
\end{aligned}
$$

where $\epsilon$ is the primitive 5 -th root of unity. Then $D_{5}$-invariant symmetric determinantal quintics can be parameterized as the determinant of the following matrices (cf. [C1]):

$$
A=\left(\begin{array}{ccccc}
0 & a_{1} y_{1} & a_{2} y_{2} & a_{2} y_{3} & a_{1} y_{4} \\
a_{1} y_{1} & a_{3} y_{2} & a_{4} y_{3} & a_{5} y_{4} & 0 \\
a_{2} y_{2} & a_{4} y_{3} & a_{6} y_{4} & 0 & a_{5} y_{1} \\
a_{2} y_{3} & a_{5} y_{4} & 0 & a_{6} y_{1} & a_{4} y_{2} \\
a_{1} y_{4} & 0 & a_{5} y_{1} & a_{4} y_{2} & a_{3} y_{3}
\end{array}\right)
$$

where $a_{1}, \ldots, a_{6}$ are parameters. Then

$$
\begin{aligned}
\operatorname{det}(A) & =\left(a_{1}^{2} a_{5}^{2} a_{6}\right) f_{1}+\left(a_{2}^{2} a_{3} a_{4}^{2}\right) f_{2} \\
& +\left(-2 a_{1}^{2} a_{4} a_{5} a_{6}-2 a_{1} a_{2} a_{5}^{3}-a_{1}^{2} a_{3} a_{6}^{2}\right) f_{3} \\
& +\left(-2 a_{2}^{2} a_{3} a_{4} a_{5}-2 a_{1} a_{2} a_{4}^{3}-a_{2}^{2} a_{3}^{2} a_{6}\right) f_{4} \\
& +\left(2 a_{1} a_{2} a_{3} a_{4} a_{6}+2 a_{1} a_{2} a_{4}^{2} a_{5}+a_{2}^{2} a_{3} a_{5}^{2}\right) f_{5} \\
& +\left(2 a_{1} a_{2} a_{3} a_{5} a_{6}+2 a_{1} a_{2} a_{4} a_{5}^{2}+a_{1}^{2} a_{4}^{2} a_{6}\right) f_{6}
\end{aligned}
$$

where

$$
\begin{aligned}
f_{1}=y_{1}^{5}+y_{4}^{5} & f_{2}=y_{2}^{5}+y_{3}^{5} \\
f_{3}=y_{1} y_{4}\left(y_{1}^{2} y_{3}+y_{2} y_{4}^{2}\right) & f_{4}=y_{2} y_{3}\left(y_{1} y_{2}^{2}+y_{3}^{2} y_{4}\right) \\
f_{5}=y_{2} y_{3}\left(y_{1}^{2} y_{3}+y_{2} y_{4}^{2}\right) & f_{6}=y_{1} y_{4}\left(y_{1} y_{2}^{2}+y_{3}^{2} y_{4}\right) .
\end{aligned}
$$

Example 8. Let $a_{1}=\cdots=a_{6}=1$ in the above $\operatorname{det}(A)$. We have the quintic surface,

$$
\Sigma: y_{1}^{5}+y_{2}^{5}+y_{3}^{5}+y_{4}^{5}+5\left(y_{1} y_{4}-y_{2} y_{3}\right)\left(y_{1} y_{2}^{2}+y_{3}^{2} y_{4}-y_{1}^{2} y_{3}-y_{2} y_{4}^{2}\right)=0 .
$$

Consider a pencil $\left|2 K_{\Sigma}\right|^{\mathbb{Z}_{5}}: \lambda y_{1} y_{4}-\mu y_{2} y_{3}$ where $(\lambda, \mu) \in \mathbb{P}^{1}$. We want to prove that every curve in this pencil is a stable curve. 
Let $\lambda=1, y_{1}=1$, and $y_{4}=\mu y_{2} y_{3}$. Consider the following three equations:

$$
\begin{aligned}
& \Sigma=0: 1+y_{2}^{5}+y_{3}^{5}+\mu^{5} y_{2}^{5} y_{3}^{5}=5(\mu-1)\left(y_{2} y_{3}^{2}+\mu^{2} y_{2}^{4} y_{3}^{3}-y_{2}^{3} y_{3}-\mu y_{2}^{2} y_{3}^{4}\right) \\
& \frac{\partial \Sigma}{\partial y_{2}} \cdot \frac{y_{2}}{5}=0: y_{2}^{5}+\mu^{5} y_{2}^{5} y_{3}^{5}=(\mu-1)\left(y_{2} y_{3}^{2}+4 \mu^{2} y_{2}^{4} y_{3}^{3}-3 y_{2}^{3} y_{3}-2 \mu y_{2}^{2} y_{3}^{4}\right), \\
& \frac{\partial \Sigma}{\partial y_{3}} \cdot \frac{y_{3}}{5}=0: y_{3}^{5}+\mu^{5} y_{2}^{5} y_{3}^{5}=(\mu-1)\left(2 y_{2} y_{3}^{2}+3 \mu^{2} y_{2}^{4} y_{3}^{3}-y_{2}^{3} y_{3}-4 \mu y_{2}^{2} y_{3}^{4}\right) .
\end{aligned}
$$

Case (1): If $\mu=1$, then $y_{2}^{5}=y_{3}^{5}=-1, y_{1}=1, y_{4}=y_{2} y_{3}$ satisfy the above three equations. After $\mathbb{Z}_{5}$-action, there are five different singular points on the curve, $\Sigma \cap\left(y_{1} y_{4}=y_{2} y_{3}\right)$.

Case (2): Assume

$$
\left\{\begin{array}{l}
\mu \neq 1 \\
y_{1}=1, y_{4}=\mu y_{2} y_{3}, \\
y_{2}=\mu y_{3}^{3} \\
\mu^{5} y_{3}^{10}=1 \\
y_{3}^{5}+1=5(\mu-1) y_{3}^{5} \mu\left(1-\mu^{2} y_{3}^{5}\right)
\end{array}\right.
$$

From this assumption, $\mu$ is the solution of the equation $\mu^{4}-14 \mu^{3}+51 \mu^{2}-14 \mu+1=$ $\left(\mu^{2}-7 \mu+1\right)^{2}=0$. Then this assumption satisfies the above three equations. For each $\mu$, we have ten different $y_{3}$ and $\mu$. Since $y_{3}$ determines $y_{2}$ and $y_{4}$, we have two curves (solutions of $\mu^{2}-7 \mu+1=0$ ) that have two singular points on each curve after $\mathbb{Z}_{5}$-action.

Case (3): Assume $\mu=0, y_{1}=1$ and $y_{4}=0$. Let $\xi$ be the 5 -th root of unity. Let $y_{3}=\xi^{i}, y_{2}=a \xi^{j}$ such that $2 i+j \equiv 0(\bmod 5), i+3 j \equiv 0(\bmod 5)$. And $a$ is the solution of $x^{2}-x-1=0$. Consider the pairs

$$
\left(\begin{array}{l}
y_{3}=1 \\
y_{2}=a
\end{array}\right) \quad\left(\begin{array}{c}
y_{3}=\xi \\
y_{2}=a \xi^{3}
\end{array}\right) \quad\left(\begin{array}{l}
y_{3}=\xi^{2} \\
y_{2}=a \xi
\end{array}\right) \quad\left(\begin{array}{c}
y_{3}=\xi^{3} \\
y_{2}=a \xi^{4}
\end{array}\right) \quad\left(\begin{array}{c}
y_{3}=\xi^{4} \\
y_{2}=a \xi^{2}
\end{array}\right) .
$$

This $y_{1} \ldots y_{4}$ satisfies the above three equations. According to an easy computation, these points satisfy the four equations: $\frac{\partial \Sigma}{\partial y_{i}}=0$ for $i=1,2,3,4$. So these points are singular points on the surface, and it is easy to check that they are node singular points.

Case (4): Let $\mu=0$ and $y_{1}=y_{4}=0$. Then $\Sigma$ change to $y_{2}^{5}+y_{3}^{5}=0$.

By the action of $\mathbb{Z}_{4}$ on $\Sigma: y_{1} \rightarrow y_{2} \rightarrow y_{4} \rightarrow y_{3}$, we have eight different singular points on $\mu=0$ or $\mu=\infty$ after $\mathbb{Z}_{5}$-action. So we have $\delta_{0}=25$ (because each node point of a surface counts as two) and $\delta_{2}=2$ after $\mathbb{Z}_{5}$-action on $\Sigma$ where $\delta_{i}$ are the standard boundary generators of Pic $\left(\overline{\mathcal{M}_{4}}\right)$. According to Lemma 4 , these are the right intersection numbers for the bicanonical fibration of $\Sigma / \mathbb{Z}_{5}$.

According to Example 8 and the irreducibility of moduli space, we obtain the following:

Lemma 9. Each curve, in the bicanonical pencil of a general $D_{5}$-invariant determinantal Godeaux surface, is a stable curve.

Consider a $D_{5}$-invariant symmetric determinantal quintic $\Sigma$. Give a twisted $D_{5}=\langle b, a \sigma\rangle$-action on a double cover $\sigma: Y \rightarrow \Sigma$. Barlow proves that the minimal resolution of $B=Y /\langle b, a \sigma\rangle$ is a surface of general type, with $p_{g}=q=0, K^{2}=1$, 
and simply connected (determinantal Barlow surface). Consider a commutative diagram

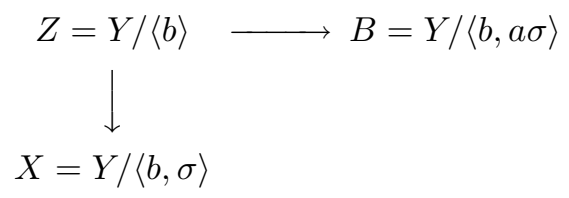

In diagram (2.1), $X$ is a $D_{5}$-invariant determinantal Godeaux surface and $B$ is a determinantal Barlow surface. According to the definition of actions, two involutions $a, \sigma$ act on the fibers of bicanonical system of $Z$. This also implies that each member of a determinantal Barlow surface is a stable curve, because the $a, \sigma$-actions preserve the fibers of the bicanonical pencil. By an easy computation and Lemma 9, we obtain the following:

Lemma 10. Each curve, in the bicanonical pencil of a general determinantal Barlow surface, is a stable curve. Let $f: S \rightarrow \mathbb{P}^{1}$ be the bicanonical pencil of a general determinantal Barlow surface. Then $\lambda \cdot \mathbb{P}^{1}=4, \delta_{0} \cdot \mathbb{P}^{1}=27$, and $\delta_{1} \cdot \mathbb{P}^{1}=\delta_{2} \cdot \mathbb{P}^{1}=0$.

\section{UnobstruCted Deformation of a DETERMinantal Barlow SURFACE}

Theorem 11. The second cohomology of the tangent sheaf of a determinantal Barlow surface is zero.

Proof. Let $\tilde{q}: \mathbb{P}^{1} \rightarrow \overline{\mathcal{M}}_{4}$ be the bicanonical pencil that comes from a $D_{5}$-invariant determinantal Godeaux surface. Through a computation that is analogous to that in the proof of Theorem 6 and considering $D_{5}$-invariant quintics, we have

$$
\tilde{q}^{*} T_{\overline{\mathcal{M}}_{4}}=\mathcal{O}(-1)^{4}+\mathcal{O}(1)^{4}+\mathcal{O}(2) .
$$

Inside of $\mathcal{O}(1)^{4}$, (+)-eigenspace of $\mathbb{Z}_{2}=D_{5} / \mathbb{Z}_{5}$ is $\mathcal{O}(1)^{2}$. Consider diagram (2.1). Let $\mathbb{P}_{X}^{1}$ be the bicanonical pencil of a $D_{5}$-invariant determinantal Godeaux surface and $\mathcal{U}_{X}$ an analytic neighborhood of $\mathbb{P}_{X}^{1}$ in $\overline{\mathcal{M}_{4}}$. Barlow construction distinguishes, for each $\{C\} \in \mathbb{P}_{X}^{1}$, a cardinality-four group of the unbranched double cover of $C$. Using this we lift $\mathbb{P}_{X}^{1} \subseteq \mathcal{U}_{X}$ to $\mathbb{P}_{Z}^{1} \subseteq \mathcal{U}_{Z}$ in $\overline{\mathcal{M}_{7, i}}=\overline{\mathcal{R}_{7}}$, the deformation of the pair $(\tilde{C}, i)$ where $\tilde{C}$ is a double cover of $C$ in $\left|2 K_{X}\right|$ and $i$ is the involution. Then Barlow's twisting a-action gives an involution of $\mathcal{N}$, taking $\mathbb{P}_{Z}^{1} \subseteq \mathcal{U}_{Z}$ to $\mathbb{P}_{Z}^{1}{ }^{\prime} \subseteq \mathcal{U}_{Z}^{\prime}$ in $\overline{\mathcal{R}_{7}}$. Let $\mathbb{P}_{B}^{1}$ be the bicanonical pencil of a determinantal Barlow surface. Then this neighborhood $\mathcal{U}_{Z}^{\prime}$ is sent to the analytic neighborhood $\mathcal{U}_{B}$ of $\mathbb{P}_{B}^{1}$ in $\overline{\mathcal{M}_{4}}(Z \rightarrow B$ in diagram (2.1)). See diagram (3.1). There are four-branching points in the step of lifting of $\mathbb{P}_{X}^{1}$ to $\mathbb{P}_{Z}^{1}$. The Prym map from $\overline{\mathcal{R}_{7}}$ to $\overline{\mathcal{M}_{4}}$ has a branch locus that is tangent to the Godeaux pencil at four points, or, in the $D_{5}$-invariant determinantal Godeaux case, has contact four at two points of the pencil. Since each point decreases one-dimensional first order deformation and four nodes are independent [L1, §3.2], the normal bundle upstairs is $\mathcal{O}^{4}+\mathcal{O}(-1)^{4}$ for a general $Z$, or, is $\mathcal{O}^{4}+\mathcal{O}(-1)^{4}$ or $\mathcal{O}(1)^{2}+\mathcal{O}(-1)^{2}+\mathcal{O}(-1)^{4}$ for the $D_{5}$-invariant case. In any case, the possible component of a negative part of the normal bundle of a determinantal Barlow surface is $\mathcal{O}(-1)$, since $a$-action preserves the normal 
bundle and the degrees do not decrease under finite morphisms.

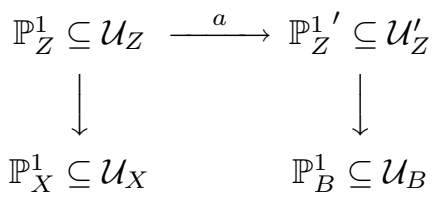

According to Lemma 5, we obtain the theorem.

\section{Hyperelliptic Members in the BiCANonical PENCiL of A DETERMinANTAL BARLOW SURFACE}

Let $X$ be a numerical Godeaux surface. Assume that the bicanonical pencil has no base components and a general member in $\left|2 K_{X}\right|$ is smooth. According to semi-positiveness and Hirzebruch-Riemann-Roch theorem, we obtain the following:

Lemma 12. 1. $f_{*}\left(p^{*} 2 K_{X}\right)=\mathcal{O}(1)$,

2. $f_{*}\left(p^{*} 3 K_{X}\right)=\mathcal{O}^{4}$,

3. $f_{*}\left(p^{*} 4 K_{X}\right)=\mathcal{O}^{4}+\mathcal{O}(2)$,

4. $f_{*}\left(p^{*} 5 K_{X}\right)=\mathcal{O}^{3}+\mathcal{O}(1)^{4}$,

5. $f_{*}\left(p^{*} 6 K_{X}\right)=\mathcal{O}^{4}+\mathcal{O}(1)^{4}+\mathcal{O}(3)$,

6. $f_{*}\left(p^{*} 7 K_{X}\right)=\mathcal{O}^{4}+\mathcal{O}(1)^{3}+\mathcal{O}(2)^{4}$,

7. $f_{*}\left(p^{*} 8 K_{X}\right)=\mathcal{O}^{4}+\mathcal{O}(1)^{4}+\mathcal{O}(2)^{4}+\mathcal{O}(4)$,

8. $f_{*}\left(p^{*} 9 K_{X}\right)=\mathcal{O}^{4}+\mathcal{O}(1)^{4}+\mathcal{O}(2)^{3}+\mathcal{O}(3)^{4}$,

where $p: S \rightarrow X$ is a blow up of the base points of $\left|2 K_{X}\right|$ and a fibration $f: S \rightarrow \mathbb{P}^{1}$ is given by the bicanonical pencil.

Since $f_{*} \omega_{S / \mathbb{P}^{1}}=\mathcal{O}(1)^{4}$ and $\left.p^{*} 3 K_{X}\right|_{C}=K_{C}$ for every fiber of $f: S \rightarrow \mathbb{P}^{1}$, we have the following lemma.

Lemma 13. 1. $f_{*} \omega_{S / \mathbb{P}^{1}}=\mathcal{O}(1)^{4}$,

2. $f_{*} \omega_{S / \mathbb{P}^{1}}^{\otimes 2}=\mathcal{O}(2)^{4}+\mathcal{O}(3)^{4}+\mathcal{O}(5)$,

3. $f_{*} \omega_{S / \mathbb{P}^{1}}^{\otimes 3}=\mathcal{O}(3)^{4}+\mathcal{O}(4)^{4}+\mathcal{O}(5)^{3}+\mathcal{O}(6)^{4}$.

In fact, $f_{*} \omega_{S / \mathbb{P}^{1}}^{\otimes i}$ for $i=1,2,3$ determine the surface. The author proves the following corollary in L1 $\$ 2.3]$.

Corollary 14. Let $S$ be a smooth projective surface with a pencil $f: S \rightarrow \mathbb{P}^{1}$ satisfying the following:

1. each fiber is a stable curve with genus 4,

2. $f_{*} \omega_{S / \mathbb{P}^{1}}=\mathcal{O}(1)^{4}$,

3. $f_{*} \omega_{S / \mathbb{P}^{1}}^{\otimes 2}=\mathcal{O}(2)^{4}+\mathcal{O}(3)^{4}+\mathcal{O}(5)$,

4. $f_{*} \omega_{S / \mathbb{P}^{1}}^{\otimes 3}=\mathcal{O}(3)^{4}+\mathcal{O}(4)^{4}+\mathcal{O}(5)^{3}+\mathcal{O}(6)^{4}$.

Then $S$ is a blow up of the base points of $\left|2 K_{X}\right|$ of a numerical Godeaux surface $X$.

The following computation of the number of hyperelliptic curves in the bicanonical pencil is mostly due to Catanese, but we approach the proof independently in final step by using numerical data of Prym curves.

Consider the quadric sequence and the cubic sequence

$$
\begin{aligned}
& 0 \rightarrow \mathcal{K}_{2} \rightarrow S^{2}\left(f_{*} p^{*} 3 K_{X}\right) \rightarrow f_{*}\left(p^{*} 6 K_{X}\right) \rightarrow \tau_{2} \rightarrow 0 \\
& 0 \rightarrow \mathcal{K}_{3} \rightarrow S^{3}\left(f_{*} p^{*} 3 K_{X}\right) \rightarrow f_{*}\left(p^{*} 9 K_{X}\right) \rightarrow \tau_{3} \rightarrow 0
\end{aligned}
$$


where $\mathcal{K}_{i}$ and $\tau_{i}$ are defined by the kernel sheaves and cokernel sheaves. By Lemma 12 , we have

$$
\begin{gathered}
0 \rightarrow \mathcal{K}_{2} \rightarrow \mathcal{O}^{10} \rightarrow \mathcal{O}^{4}+\mathcal{O}(1)^{4}+\mathcal{O}(3) \rightarrow \tau_{2} \rightarrow 0 \\
0 \rightarrow \mathcal{K}_{3} \rightarrow \mathcal{O}^{20} \rightarrow \mathcal{O}^{4}+\mathcal{O}(1)^{4}+\mathcal{O}(2)^{3}+\mathcal{O}(3)^{4} \rightarrow \tau_{3} \rightarrow 0 .
\end{gathered}
$$

By a theorem in [C2 or $\mathrm{Mi}],\left|3 K_{X}\right|$ has no base points for a determinantal Barlow surface. For a determinantal Barlow surface, the intersection number with $\delta_{1}, \delta_{2}$ is zero by Lemma 10 . Furthermore, nonhyperelliptic fibers are 3-connected, therefore the support of the torsion sheaf consists of the points of hyperelliptic fibers (cf. Lemma 2.1 in $[\mathrm{CP}]$ ). According to Lemma 2.1 and Proposition 2.3 in [CP] (suggestion of the referee), we have

$$
\begin{aligned}
& \text { length } \tau_{2}=\sum_{i=1}^{m} 2 \ell_{i} \\
& \text { length } \tau_{3}=\sum_{i=1}^{m} 5 \ell_{i}
\end{aligned}
$$

where $m$ is the number of hyperelliptic members and $\ell_{i}$ is the contact number of pencils with hyperelliptic locus. The natural surjective homomorphism $S^{2}\left(f_{*} p^{*} 3 K_{X}\right) \otimes$ $f_{*} p^{*} 3 K_{X} \rightarrow S^{3}\left(f_{*} p^{*} 3 K_{X}\right)$ induces a homomorphism $\mathcal{K}_{2}^{4} \rightarrow \mathcal{K}_{3}$. This homomorphism is injective and the cokernel is invertible because it is torsion free (it is done in the proof of Theorem 2.5 in $\mathrm{CP}$ by embedding it in a locally free sheaf). Therefore we have the following relation between $\mathcal{K}_{2}$ and $\mathcal{K}_{3}$,

$$
0 \rightarrow \mathcal{K}_{2}^{4} \rightarrow \mathcal{K}_{3} \rightarrow \mathcal{O}(a) \rightarrow 0 .
$$

According to (4.1) and (4.2), the possible number of hyperelliptic curves (including multiplicity) $\sum_{i=1}^{m} \ell_{i}$ in (4.2) is one of $\{0,1,2,3\}$.

If we denote $\Theta_{\text {null }}$ with $\lambda, \delta_{i}$ in $\overline{\mathcal{M}_{4}}$, then

$$
\Theta_{\text {null }}=34 \lambda-4 \delta_{0}-14 \delta_{1}-18 \delta_{2}[\mathrm{Te} \text {. }
$$

According to Lemma $10, \mathbb{P}^{1} . \Theta_{\text {null }}=28$. Next, to see the multiplicity of a hyperelliptic curve $C$ of genus four as a point of $\Theta_{\text {null }}$ in $\mathcal{M}_{4}$, let $P_{1} \ldots P_{10}$ be ramification points of $C \rightarrow \mathbb{P}^{1}$. Then $g_{2}^{1}+P_{i}$ gives even theta characteristic with $h^{0} \geq 2$. So the multiplicity is ten for a hyperelliptic curve. Therefore, the possible number of hyperelliptic curves (including multiplicity) is one of $\{0,1,2\}$.

Let $\mathcal{K}=\left(\mathcal{K}_{2}\right)^{*}$, and consider the degree of $\mathcal{K}$. By (4.1) and (4.2), the degree of $\mathcal{K}$ can be 3,5 , and 7 depending on the number of hyperelliptic curves. Consider a symmetric matrix of the coefficients of quadrics in $\mathbb{P}^{3}$ corresponding to

$$
\lambda_{0}^{n} Q_{1}(x)+\lambda_{0}^{n-1} \lambda_{1} Q_{2}(x)+\cdots+\lambda_{1}^{n} Q_{n+1}(x)=0
$$

for $\left(\lambda_{0}, \lambda_{1}\right) \in \mathbb{P}^{1}, x \in \mathbb{P}^{3}$ and $n=\operatorname{deg} \mathcal{K}$. The determinant of this matrix is a polynomial of degree $4 n$ in $\mathbb{P}^{1}$, and that degree is the same as the number of singular quadrics. According to $\mathbb{P}^{1} . \Theta_{\text {null }}=28$ and the degree of $\mathcal{K}$, we obtain the following:

Lemma 15. Let $h=\sum_{i=1}^{m} \ell_{i}$ in (4.2). Then the following cases are the possibilities of a determinantal Barlow surface:

1. $h=2, \operatorname{deg} \mathcal{K}=3$ and $4 n=12$,

2. $h=1, \operatorname{deg} \mathcal{K}=5$ and $4 n=20$, 
3. $h=0, \operatorname{deg} \mathcal{K}=7$ and $4 n=28$.

If $C$ is a hyperelliptic curve, the number of singular quadrics for $C$ is counted as 2 (because the hyperelliptic curve is the double twisted cubic curve inside of the singular quadric cone). So then, the above three cases are compatible with the intersection number with $\Theta_{\text {null }}=28$.

For a general member $C$ (without passing through 4 nodes which are the branch points of a double covering of a determinantal Godeaux surface or a determinantal Barlow surface [Ba]) in the bicanonical pencil of either a determinantal Godeaux surface or a determinantal Barlow surface, there is a distinguished double cover $(\tilde{C} / C)$ without branch points. In a determinantal Godeaux surface, we have two special members of $\left|2 K_{X}\right|$, two union of genus two curves that come from $\delta_{2}=2$. Let $C$ be one of these special members. Since the double cover $(\tilde{C} / C)$ has no fixed points (for general determinantal Godeax surfaces, these two special members do not pass through 4 nodes), $\tilde{C}=\tilde{C}_{1}+\tilde{C}_{2}$ such that $\tilde{C}_{1} \cdot \tilde{C}_{2}=$ two points. Let $C^{\prime}$ be a corresponding curve in a determinantal Barlow surface. Then $C^{\prime}$ is an irreducible curve with one node. This explains the reason for the number $\delta_{2}=2$ changing to $\delta_{0}$ when we change from a determinantal Godeaux surface to a determinantal Barlow surface (see diagram (2.1)).

The associated Prym curve $D=\operatorname{Prym}(\tilde{C}, C)$ is the Jacobian of a curve of genus three. Thus our bicanonical pencil induces a mapping, $q: \mathbb{P}^{1} \rightarrow \overline{\mathcal{M}_{3}}$. Let $\lambda, \delta_{0}, \delta_{1}$ be the standard generators of Pic $\overline{\mathcal{M}_{3}}$.

The lifting of the bicanonical pencil of a determinantal Godeaux or a determinantal Barlow surface into a double covering $Z$ induces a morphism $\tilde{f}: \tilde{Z} \rightarrow \mathbb{P}^{1}$ where $\tilde{Z}$ is an 8-point blow up of $Z$ (In general, the base points of the bicanonical pencil are not the branch points of double covering). According to the semi-positiveness theorem of curves (Theorem of Fujita in $[\mathrm{Fu}]$ ) and numerical data of $\tilde{Z}$, we obtain

$$
\tilde{f}_{*} \omega_{\tilde{Z} / \mathbb{P}^{1}}=\mathcal{O}(1)^{7} .
$$

Since the push down of the (+)-eigenspace of $\sigma$ or $a \sigma$ is $\mathcal{O}(1)^{4}$, the (-)-eigenspace of $\sigma$ or $a \sigma$ is $\mathcal{O}(1)^{3}$ (see diagram (2.1)).

Consider Prym curve $D \in \overline{\mathcal{M}_{3}}$ at two special points induced by $\delta_{2}=2$ with the bicanonical pencil of a determinantal Godeaux surface. Then

$$
1 \rightarrow \mathbb{C}^{*} \rightarrow \mathrm{Pic}^{\circ} D \rightarrow \prod_{i=1}^{2} \mathrm{Pic}^{\circ} D_{i} \rightarrow 1
$$

where $\operatorname{Pic}^{\circ} D_{i}=$ elliptic curve. So $D=D_{1}+D_{2}$, such that $D_{i}$ is an elliptic curve and they meet two points. Let $D^{\prime}=\operatorname{Prym}\left(\tilde{C}, C^{\prime}\right)$. Then $D^{\prime}$ is a smooth curve of genus three. If $C$ is a curve of $p_{a}(C)=4$ with one node, and if the involution $i: \tilde{C} \rightarrow C$ interchanges two nodes of $\tilde{C}$, then the corresponding Prym curve $D$ is a curve of $p_{a}(C)=3$ with one node. And if the involution fixes the node of $\tilde{C}$, then Prym curve $D$ is a smooth curve of genus three. Consider the partition $\delta_{0}=27=2$ (related to $\delta_{2}=2$ for a determinantal Godeaux surface) +8 (related to 4 branch points) +17 for a determinantal Barlow surface. By the above arguments we obtain the following:

Lemma 16. Let $\lambda, \delta_{0}, \delta_{1}$ be the standard generators of Pic $\overline{\mathcal{M}_{3}}$. Then we have the following intersection data with $q\left(\mathbb{P}^{1}\right)$ for a determinantal Barlow surface

$$
\lambda=3, \delta_{0}=17, \delta_{1}=0 .
$$


Theorem 17. For a determinantal Barlow surface, the number of hyperelliptic curves (it can be a denegenerating curve of a hyperelliptic curve) in the bicanonical pencil is two.

Proof. Consider the Prym construction. According to Lemma 16, we have the following intersection data with the associated Prym curves of the bicanonical pencil: $\lambda=3, \delta_{0}=17$ and $\delta_{1}=0$. Also $\Theta_{\text {null }}$ in $\overline{\mathcal{M}_{3}}$, is provided by

$$
\Theta_{\text {null }}=9 \lambda-\delta_{0}-3 \delta_{1}[\mathrm{Te}]
$$

For a determinantal Barlow surface case, $q\left(\mathbb{P}^{1}\right) \cdot \Theta_{\text {null }}=10$. In $\overline{\mathcal{M}_{3}}$, the multiplicity of a hyperelliptic curve in $\Theta_{\text {null }}$ is one. Furthermore, if the original curve has even theta characteristic with a positive dimension, then the Prym curve does also. So, of the above three cases, only the case of $h=2$ is possible for a determinantal Barlow surface.

Remark 18. Theorem 17 is also obtained by [CP in a more general setting via calculation of the syzygies for the canonical ring and help of the Macaulay program. According to Theorem 17, blowing up at four base points of the bicanonical pencil of a general determinantal Barlow surface has the presentation $(2,3)$ and $(3,0)$. So we have a divisor of type $(3,0)$ inside a hypersurface of bidegree $(2,3)$. There is added significance to the two hyperelliptic curves when we consider a compactification of a family of determinantal Barlow surfaces. The role of two hyperelliptic members in the bicanonical pencil is the same as the role of two special members from $\delta_{2}=2$ in the bicanonical pencil of a determinantal Godeaux surface in [L2].

\section{REFERENCES}

[AC] E. Arbarello and M. Cornalba, The Picard groups of the moduli spaces of curves, Topology 26 (1987), 153-171. MR 88e:14032

[Ba] R. Barlow, A simply connected surface of general type with $p_{g}=0$, Invent. Math. 79 (1985), 293-301. MR 87a:14033

[B1] A. Beauville, Variétés de Prym et jacobiennes intermédiaires, Ann. Sci. École Norm. Sup. 10 (1977), 309-391. MR 57:12532

[B2] A. Beauville, L'application canonique pour les surfaces de type général, Invent. Math. 55 (1979), 121-140. MR 81m:14025

[B3] A. Beauville, L'inégalités $p_{g} \geq 2 q-4$ pur les surfaces de type général, Appendix to O. Debarre: Inégalités numérique pour les surfaces de type général, Bull. Soc. Math. Fr. 110 (1982), 319-346. MR 84f:14026

[C1] F. Catanese, Babbage's conjecture, contact of surfaces, symmetric determinantal varieties and applications, Invent. Math. 63 (1981), 433-465. MR 83c:14026

[C2] F. Catanese, Pluricanonical mapping of surfaces with $K^{2}=1,2, q=p_{g}=0$, C.I.M.E. 1977 Algebraic Surfaces, Liguori, Napoli (1981), 249-266.

[CL] F. Catanese and C. Le Brun, On the scalar curvature of Einstein manifolds, Math. Res. Letters 4 (1997), 843-854. MR 98k:53057

[CP] F. Catanese and R. Pignatelli, On simply connected Godeaux surfaces, Preprint (1999).

[Fu] T. Fujita, On Kähler fiber spaces over curves, J. Math. Soc. Japan 30 (1978), 779-794. MR 82h:32024

[HM] J. Harris and D. Mumford, On the Kodaira dimension of the moduli space of curves, Invent. Math. 67 (1982), 23-88. MR 83i:14018

[Ko] J. Kollár, Rational curves on algebraic varieties, Springer-Verlag, 1996. MR 98c:14001

[L1] Y. Lee, Degeneration of numerical Godeaux surfaces, Ph.D. Thesis, University of Utah (1997).

[L2] Y. Lee, A compactification of a family of determinantal Godeaux surfaces, Trans. Amer. Math. Soc. 352 (2000), 5013-5023. CMP 98:13 
[Mi] Y. Miyaoka, Tricanonical maps of numerical Godeaux surfaces, Invent. Math. 34 (1976), 99-111. MR 53:13236

[Re] M. Reid, Surfaces with $p_{g}=0, K^{2}=1$, J. Fac. Sc. Univ. of Tokyo IA 25, No 1 (1978), 75-92. MR 80h:14018

[Te] M. Texidor, The divisor of curves with a vanishing theta-null, Compositio Math. 66 (1988), $15-22$.

Korea Institute for Advanced Study, 207-43 Cheongryangri-dong, Dongdaemun-gu, SEOUl 130-012, Korea

Current address: Department of Mathematics, Sogang University, Sinsu-dong, Mapo-gu, Seoul 121-742, Korea

E-mail address: ynlee@ccs.sogang.ac.kr 